\title{
Impact of Behavioural Changes in Mosquito Feeding on Malaria Invasion: A Model-Based Approach
}

\section{Sungchan Kim}

Pusan National University

\section{Yongkuk Kim}

Kyungpook National University

Byul Nim Kim

Kyungpook National University

\section{A Masud}

Pusan National University

II Hyo Jung ( $\nabla$ ilhjung@pusan.ac.kr)

Pusan National University College of Natural Science https://orcid.org/0000-0002-6086-5124

\section{Research}

Keywords: branching process, individual-based stochastic simulation algorithm, transmission heterogeneity, vector-bias

Posted Date: August 25th, 2020

DOl: https://doi.org/10.21203/rs.3.rs-62181/v1

License: (c) (i) This work is licensed under a Creative Commons Attribution 4.0 International License. Read Full License 
2

3

\section{${ }_{5}^{4}$ Impact of Behavioural Changes in Mosquito}

${ }_{7}^{6}$ Feeding on Malaria Invasion: A Model-Based

${ }_{9}^{8} A$ pproach

${ }^{10}$ Sungchan Kim ${ }^{1,2}$, Yongkuk Kim ${ }^{3}$, Byul Nim Kim ${ }^{4}, \mathrm{M} \mathrm{A} \mathrm{Masud}{ }^{2,5, *}$ and II Hyo Jung ${ }^{1,2, *}{ }^{10}$ 11

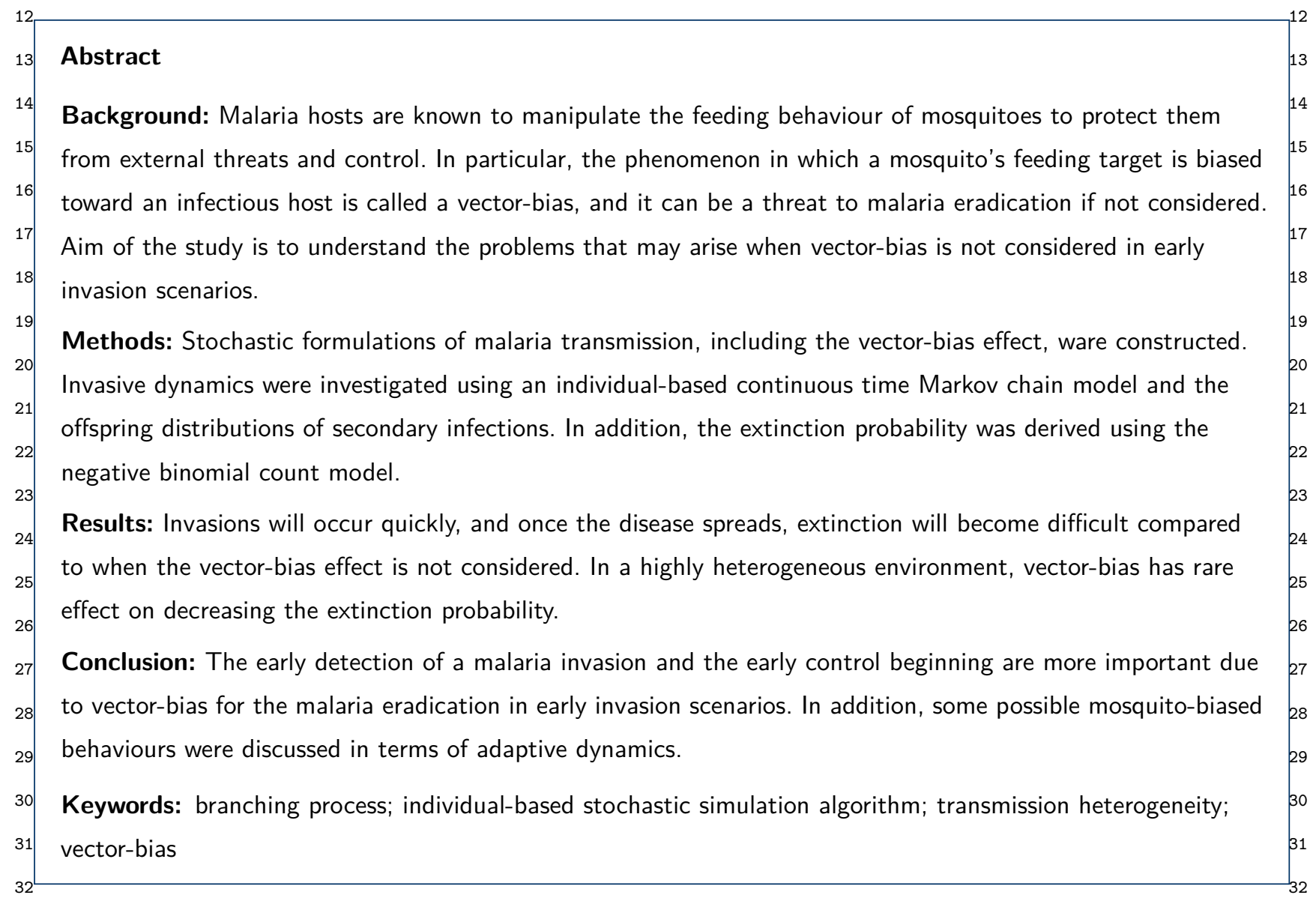

33

\section{${ }_{34}$ Introduction}

${ }_{35}$ The adaptive host manipulation hypothesis [1] states ${ }_{36}$ that parasites evolve themselves to control specific as${ }_{37}$ pects of their host's behaviour and thereby enhance

Full list of author information is available at the end of the article

${ }^{38}{ }^{*}$ Corresponding authors: M A Masud (ma.masud@northsouth.edu), and II

${ }^{39}$ Hyo Jung (ilhjung@pusan.ac.kr) the frequency of their encounters with suitable hosts ${ }_{34}$ and thus, the rate of transmissibility of a parasite as $_{35}$ well [2]. Such manipulation by parasites has also been ${ }_{36}$ found in several cases of malaria outbreaks. For ex- $_{37}$ ample, a mosquito finding a host to feed on is influenced by the host odour and olfactory cues $[3,4]_{39}$ 
${ }^{1}$ attributes that are exploited by parasites to attract ${ }^{2}$ mosquitos [3, 4, 5, 6, 7]. During the 1980s, an ex${ }^{3}$ periment was conducted using mice [8, 9], hens [10], ${ }^{4}$ and lambs [11], in which mosquitoes were found to ${ }^{5}$ feed preferentially on infected hosts. A decade earlier, ${ }^{6}$ a similar result was found in the case of malaria in ${ }^{7}$ a human host [12], in which it was determined that ${ }^{8}$ malaria-infected humans have a greater attractiveness ${ }^{9}$ to mosquitoes, a phenomenon that was later called ${ }^{10}$ vector-bias [13], and more evidences of mosquito's feed${ }^{11}$ ing bias toward Plasmodium-infected vertebrates were ${ }^{12}$ found $[14,15,16,17,18,19]$. Such studies encouraged ${ }^{13}$ thinking over whether the parasites manipulate their ${ }^{14}$ hosts to increase their probability of survival or not. 15

16 Such manipulation of mosquitoes by malaria para${ }^{17}$ sites cause significant difficulties in predicting or con${ }^{18}$ trolling malaria $[20,21]$. Therefore, the mosquito feed${ }^{19}$ ing behaviour is important in epidemiological stud${ }^{20}$ ies of malaria transmission. Numerous modelling at${ }^{21}$ tempts have been made to analyse or quantify the ef${ }^{22}$ fects of mosquitoes on the changes in feeding behaviour ${ }^{23}[13,22,23,24]$. The first mathematical model, which ${ }^{24}$ dealt with mosquito behaviour increasing the total bit${ }^{25} \mathrm{ing}$ rate (the total biting rate of susceptible and in${ }^{26}$ fected mosquitoes), proved to increase the equilibrium ${ }^{27}$ infection level during endemic equilibrium, whereas in${ }^{28}$ fected host preference was found to exert both increas${ }^{29}$ ing and decreasing effects [22]. Discussions regarding ${ }^{30} \mathrm{impact}$ of the manipulation of mosquitoes by malaria ${ }^{31}$ parasites to the transmission probability of malaria dis${ }^{32}$ eases was presented by Cator et al. [25, 26]. In [13], the ${ }^{33}$ authors presented a vector-bias model and concluded ${ }^{34}$ that mosquitoes are most preferred by a high preva${ }^{35}$ lence of the parasites. Although both positive and neg${ }^{36}$ ative effects were reported quantitatively, no argument ${ }^{37}$ for this dual role has been presented, but was recently ${ }^{38}$ clarified in [27]. It was shown that mosquito encoun${ }^{39}$ ters with susceptible hosts are as likely as mosquito encounters with infected hosts, but that bites occur ${ }^{1}$ in infected hosts with a higher probability. Thereafter, ${ }^{2}$ numerous studies on vector-bias were conducted, with $^{3}$ a focus on the periodicity of mosquito abundance [28], time-delay [29], fractional-order [30], spatial structure ${ }^{5}$ [31], and real-world application [32]. It can be seen that ${ }^{6}$ the aforementioned studies dealing with the modelling ${ }^{7}$ of vector-bias focused on the impacts of the long-term ${ }^{8}$ behavioural dynamics.

However, there have been few studies on how vector ${ }^{10}$ bias provides momentum for the spread of the disease ${ }^{11}$ during the initial stages of malaria transmission. Moti- ${ }^{12}$ vated by this, in this paper, we investigated the impact ${ }^{13}$ of vector-bias at the initial stages of Plasmodium falci $i^{14}$ parum malaria transmission using a stochastic method, ${ }^{15}$ by comparing the dynamics when considering vector ${ }^{16}$ bias and when not considering it. Mainly, malaria inva $-{ }^{17}$ sion and extinction were studied. Since early dynam ${ }^{18}$ ics such as disease invasion are known to be better ${ }^{19}$ understood in stochastic models than in deterministic ${ }^{20}$ models $[33,34]$, and are also sensitive to changes in $^{21}$ the behaviour of each individual, an individual-based ${ }^{22}$ continuous time Markov chain model of the underly- ${ }^{23}$ ing approach introduced in this study was constructed. ${ }^{24}$ The suggested formulation allows applying Gillespie's ${ }^{25}$ stochastic simulation when considering the biased be $^{26}$ haviour of each mosquito $[35,36,37]$. To consider the ${ }^{27}$ transmission heterogeneity and consequent extinction ${ }^{28}$ probabilities, the offspring distribution along the nega ${ }^{29}$ tive binomial count model was calculated. Finally, the ${ }^{30}$ impact of vector-bias on malaria control was discussed. ${ }^{31}$

Materials and Methods 33 Underlying model

The host-vector model developed by Kim et. al. ${ }^{35}$ [27] deals with the effects of behavioural changes of ${ }^{36}$ mosquitos on the epidemic of Plasmodium falciparum $^{37}$ malaria, where the host population is divided into ${ }^{38}$ susceptible $\left(S_{h}\right)$, infectious $\left(I_{h}\right)$ and recovered $\left(R_{h}\right)^{39}$ 
${ }^{1}$ classes; and the mosquito population is divided into ${ }^{2}$ susceptible $\left(S_{v}\right)$, and infectious $\left(I_{v}\right)$ classes:

3

$4 \quad \frac{\mathrm{d} S_{h}(t)}{\mathrm{d} t}=\mu_{h} N-b p_{h} \frac{S_{h}(t)}{\lambda I_{h}(t)+\left(N-I_{h}(t)\right)} I_{v}(t)$

5

6

6

7

8

10

10

11

12

13

14

15

16

${ }^{17}$ Here, $1 / \gamma$ is the average infectious period of the host.

${ }^{18} \mathrm{We}$ consider the birth and death rates to be the same ${ }^{19}$ for both the host and the mosquito, as symbolized by ${ }^{20} \mu_{h}$ for the host and $\mu_{v}$ for the mosquito. In addition, ${ }^{21} \xi$ is the per capita rate of the loss of immunity of the ${ }^{22}$ recovered host, and $p_{h}$ and $p_{v}$ are the probability of ${ }^{23}$ mosquito-to-human and human-to-mosquito transmis${ }^{24}$ sion of a disease, respectively, for each bite. $\lambda$ is called ${ }^{25}$ a bias parameter. Notably, $\lambda=1$ means that there ${ }^{26}$ is no vector-bias and the vector-bias increases as the ${ }^{27}$ value of $\lambda>1$ increases. The total human population ${ }^{28} N=S_{h}(t)+I_{h}(t)+R_{h}(t)$ and mosquito population ${ }^{29} M=S_{v}(t)+I_{v}(t)$ are constants.

30 The basic reproduction number, which is the average ${ }^{31}$ number of secondary infected hosts generated by a sin${ }^{32}$ gle infected host introduced under a wholly susceptible ${ }^{33}$ state [38], is given by the following:

34 ${ }_{36}^{35} \quad R_{0}=\sqrt{\frac{b p_{h}}{\mu_{v}}} \sqrt{\frac{\lambda M b p_{v}}{N\left(\mu_{h}+\gamma\right)}} \equiv \sqrt{\lambda} \times c$,

${ }^{37}$ where $c=\sqrt{b^{2} p_{h} p_{v} M / N\left(\mu_{h}+\gamma\right)}$ is a constant. It ${ }^{38}$ should be noted that $\lambda$ increases the basic reproduc${ }^{39}$ tion number and enforces a breakout.
Distribution of a bias parameter

1 The estimated parameter $\lambda$ were fitted to a Weibull ${ }^{2}$ distribution with the probability density function 3

$$
f_{\lambda}(\varphi)=\left(\frac{\beta}{\alpha}\right) \times\left(\frac{\varphi}{\alpha}\right)^{\beta-1} \exp \left(-\left(\frac{\varphi}{\alpha}\right)^{\beta}\right) .
$$

where the scale parameter $\alpha=4.34$ and the shape $_{7}$ parameter $\beta=0.83$ (i.e., the expected value of $\lambda, \bar{\lambda}_{8}$ is 4.78 and the coefficient of variation is 0.58 ) in $[27]_{9}$ based on the assay conducted in [12]. In Figure 1, the ${ }_{10}$ suitability of a Weibull distribution fitting of the bias ${ }_{11}$ parameter was demonstrated (see Figure 1 in [27]).

Figure 1 Weibull probability plot of bias parameter: the probability density function of Weibull distribution is given by (2) with $\alpha=4.34$ and $\beta=0.83$.

Continuous time Markov chain model formulation and 18 individual-based stochastic simulation algorithm 19 Based on the underlying model (1), we formulated a2o continuous time Markov chain (CTMC) model. We de-21 fine the discrete random variables $S_{h}, I_{h}, R_{h}, S_{v} \operatorname{and}_{22}$ $I_{v}$ satisfying

$$
\begin{array}{ll}
S_{h}(t), I_{h}(t), R_{h}(t) \in\{0,1, \cdots, N\}, & 24 \\
S_{v}(t), I_{v}(t) \in\{0,1, \cdots, M\}, & 26
\end{array}
$$

and denote $X(t)=\left(S_{h}(t), I_{h}(t), R_{h}(t), S_{v}(t), I_{v}(t)\right)_{28}^{27}$ where $t \in[0, \infty)$. We denote

$p_{\left(s_{h}, i_{h}, r_{h}, s_{v}, i_{v}\right) \rightarrow\left(s_{h}+\Delta s_{h}, i_{h}+\Delta i_{h}, r_{h}+\Delta r_{h}, s_{v}+\Delta s_{v}, i_{v}+\Delta i_{v}\right)}(\Delta \ell)$

31

as the transition probability associated with the 2 stochastic process for $\Delta t>0$, not fixed but the du-33 ration at which something happens next, which is de-34 fined as follows:

$$
\begin{aligned}
& 35 \\
& 36 \\
& 37 \\
& 38
\end{aligned}
$$


Figure 2 Description of the individual-based CTMC model and its simulation algorithm.

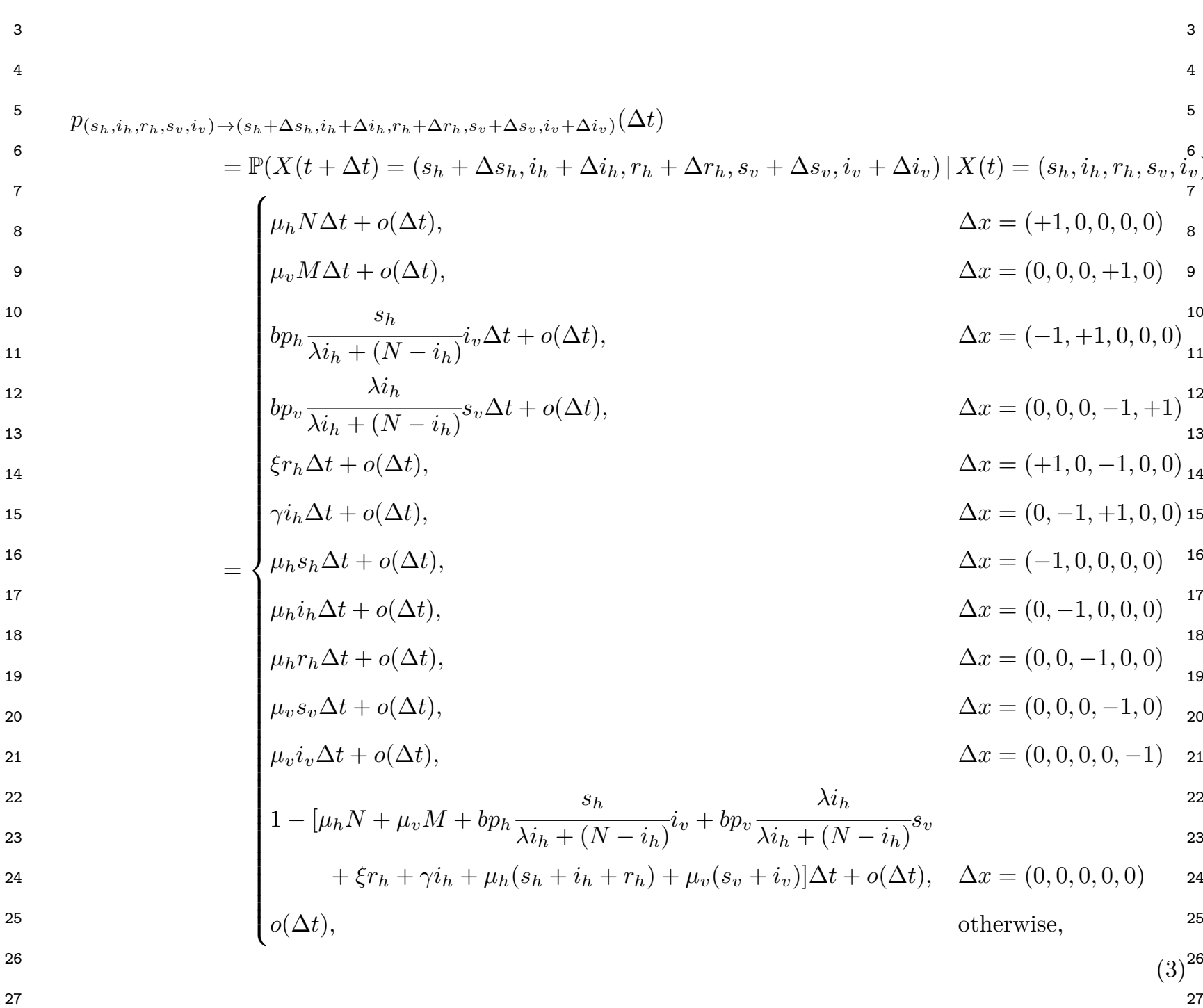

28 where $\Delta x=\left(\Delta s_{h}, \Delta i_{h}, \Delta r_{h}, \Delta s_{v}, \Delta i_{v}\right)$. The tran${ }^{29}$ sition probabilities are dependent upon the time be${ }^{30}$ tween events $\Delta t$, not on the time $t$. The time $t+\Delta t$ at ${ }^{31}$ which something happens next follows an exponential ${ }^{32}$ distribution with a parameter equal to the sum of all ${ }^{33}$ process rates.

34

35 The CTMC model (3) can be simulated using 36Gillespie's stochastic simulation algorithm. This algo37rithm is one of the most practical stochastic numerical 38simulation algorithms [35, 36, 37]. The idea behind ${ }_{39}$ Gillespie's algorithm is that it first determines when the next event will happen. Suppose that the current ${ }^{28}$ time is $t$. Second, it determines the event that is next ${ }^{29}$ to occur by randomly applying the probability follow- 30 ing the processing rates. As we showed in the previous ${ }^{31}$ section, the experimental results indicate that the $\mathrm{bi}^{-32}$ ased behaviours of each mosquito differ significantly. ${ }^{33}$ Following this, we consider a refined model (3) which ${ }^{34}$ assumes that the bias parameter of each mosquito is ${ }^{35}$ different. Motivated by [39], we extract and simulate ${ }^{36}$ sets of random bias parameters, s, for each mosquito 37 using the Monte-Carlo procedure, to allow different ${ }^{38}$ 
${ }^{1}$ bias parameter values to be assigned to each mosquito.

${ }^{2}$ From these procedures, we can divide the two events

${ }^{3}$ of human infection and mosquito infection, into $i_{v}$ in-

${ }^{4}$ dependent sub-events of human infection and $s_{v}$ inde${ }^{5}$ pendent sub-events of mosquito infection. This model

${ }^{6}$ is called as individual-based CTMC model. Table 1

${ }^{7}$ shows a summary of the events of the individual-based

${ }^{8} \mathrm{CTMC}$ model. A detailed description of the simulation

${ }^{9}$ procedure of the model is shown in Figure 2.

10 Because $\lambda$ is fitted with a Weibull distribution, the

${ }^{11}$ randomly selected bias parameter is given as 12

$13 \quad \lambda_{r_{3}}=\alpha\left(\ln \left(1 / r_{3}\right)\right)^{\beta}$

14

for the random number $r_{3}$, following a uniform distri15

bution, $\operatorname{Unif}(0,1)$. This iteration is repeated until the

${ }^{16}$ final limit is reached or there is no reaction in the sys17 tem. 18

${ }^{19}$ Branching process formulation of the underlying model

${ }^{20}$ Gillespie's stochastic simulation algorithm is suitable

${ }^{21}$ for depicting the profile or distribution of a popula-

${ }^{22}$ tion over time, although it is not possible to show the ${ }^{23}$ extinction probability and offspring distribution. We

${ }^{24}$ therefore consider the branching process model [40], ${ }^{25}$ known as the Galton-Watson process. Instead of a dis${ }^{26}$ crete branching process, a continuous branching pro${ }^{27}$ cess is suitable to deal with an infectious disease model. ${ }^{28} \mathrm{~A}$ general branching process with a finite individual ${ }^{29}$ life span dies out if and only if the embedded Galton${ }^{30}$ Watson process of the generations dies out. Thus, ${ }^{31}$ the extinction probability for the Galton-Watson pro${ }^{32}$ cesses is established in the same way for any branching ${ }^{33}$ process, in spite of the different time-to-extinction val${ }^{34} \mathrm{ues}$, because generations tend to spread out in chrono${ }^{35}$ logical time, unless reproduction and survival are sea${ }^{36}$ sonal [41]. Thus, we use the Galton-Watson process to ${ }^{37}$ estimate the extinction probability.

$38 Z_{n}$ is a random variable that denotes the number ${ }^{39}$ of individuals for each generation $n$. Here, a genera-

tion indicates the mean infection period for one person. ${ }^{1}$ When the outbreak is limited, it is assumed that one ${ }^{2}$ infection occurs in a wholly susceptible human popu- ${ }^{3}$ lation, $Z_{0}=1$, and that the offspring distribution of ${ }^{4}$ each person in each generation is an independent and ${ }^{5}$ identically distributed random variable, $Z_{1}$. Applying ${ }^{6}$ $q_{k}=\mathbb{P}\left(Z_{1}=k\right)$, the extinction probability is then de- ${ }^{7}$ fined as the smallest fixed point of $G_{Z_{1}}$, that is, the ${ }^{8}$ extinction probability is the smallest solution within ${ }^{9}$ $[0,1]$ for

10

$$
G_{Z_{1}}(x)=x
$$

where $G_{Z_{1}}$ is the probability generating function of $q_{k}{ }_{14}^{13}$

Next, we define the offspring distribution $q_{k}$. In general, the Poisson distribution is used extensively for ${ }^{15}$ counting, although the Poisson model cannot represent an overdispersion of the data. Hence, we use the negative binomial counting model $[42,43]$ for considering the heterogeneity of the population. The model is derived as follows: let $Z_{1} \mid \nu \sim \operatorname{Poisson}(\nu)$ and assume $\nu \sim \operatorname{Gamma}\left(R_{0} / s, s\right)$, i.e., $\nu$ is Gamma distributed ${ }^{21}$ with dispersion parameter $s$ with the dispersion parameter $s$ with a mean $R_{0}$. Then, a simple calculation yields

$$
q_{k}=\frac{\Gamma(s+k)}{k ! \Gamma(s)}\left(\frac{R_{0}}{s+R_{0}}\right)^{k}\left(\frac{s}{s+R_{0}}\right)^{s}, \quad \begin{array}{r}
26 \\
28
\end{array}
$$

and when considering the distribution of the bias pa- ${ }_{29}$ rameter, $f_{\lambda}$,

$$
q_{k}=\int_{0}^{\infty} \frac{\Gamma(s+k)}{k ! \Gamma(s)}\left(\frac{R_{0}}{s+R_{0}}\right)^{k}\left(\frac{s}{s+R_{0}}\right)^{s} f_{\lambda}(\varphi) 31 \varphi
$$

is obtained, where $\Gamma$ is the Gamma function [44]. Note ${ }^{35}$ that the offspring distribution $Z_{1}$ is distributed as $\mathrm{a}^{36}$ negative binomial, i.e., $Z_{1} \sim \operatorname{NegBin}\left(\frac{s}{s+R_{0}}, s\right)$ with ${ }^{37}$ variance $R_{0}\left(1+R_{0} / s\right)$. The dispersion parameter $s^{38}$ determines the level of variation in the number of ${ }^{39}$ 
${ }_{1}$ Table 1 Definition of events in the individual-based CTMC model, where $\Delta X(t)$ denotes $\Delta X(t)=X(t+\Delta t)-X(t)$.

\begin{tabular}{|c|c|c|c|c|}
\hline 2 & Event No. & Event description & Changes, $\Delta X(t)^{T}$ & Probability \\
\hline 3 & $(E 1)$ & Birth of human & $(+1,0,0,0,0)$ & $\mu_{h} N \Delta t+o(\Delta t)$ \\
\hline 4 & $(E 2)$ & Birth of mosquito & $(0,0,0,+1,0)$ & $\mu_{v} M \Delta t+o(\Delta t)$ \\
\hline 6 & $(E 3-k)$ & Human infection & $(-1,+1,0,0,0)$ & $\begin{array}{l}b p_{h} \frac{s_{h}}{N+\left(\lambda_{k}-1\right) i_{h}} \Delta t+o(\Delta t), \text { for } k= \\
1,2, \cdots, i_{v}\end{array}$ \\
\hline 8 & $(E 4-k)$ & Mosquito infection & $(0,0,0,-1,+1)$ & $\begin{array}{l}b p_{v} \frac{\lambda_{k} i_{h}}{N+\left(\lambda_{k}-1\right) i_{h}} \Delta t+o(\Delta t), \text { for } k= \\
1,2, \cdots, s_{v}\end{array}$ \\
\hline & $(E 5)$ & Loss of immunity of human & $(+1,0,-1,0,0)$ & $\xi r_{h} \Delta t+o(\Delta t)$ \\
\hline & $(E 6)$ & Recovery of human & $(0,-1,+1,0,0)$ & $\gamma i_{h} \Delta t+o(\Delta t)$ \\
\hline & $(E 7)$ & Death of susceptible human & $(-1,0,0,0,0)$ & $\mu_{h} s_{h}+o(\Delta t)$ \\
\hline & $(E 8)$ & Death of infectious human & $(0,-1,0,0,0)$ & $\mu_{h} i_{h}+o(\Delta t)$ \\
\hline & $(E 9)$ & Death of recovered human & $(0,0,-1,0,0)$ & $\mu_{h} r_{h}+o(\Delta t)$ \\
\hline 15 & $(E 10)$ & Death of susceptible mosquito & $(0,0,0,-1,0)$ & $\mu_{v} s_{v}+o(\Delta t)$ \\
\hline 16 & $(E 11)$ & Death of infectious mosquito & $(0,0,0,0,-1)$ & $\mu_{v} i_{v}+o(\Delta t)$ \\
\hline
\end{tabular}

${ }^{18}$ secondary infections. The heterogeneity increases as ${ }^{19} s<<1[42,45]$. Note that this model includes the Pois${ }^{20}$ son model $(s \rightarrow \infty)$. That is, the smaller the disper${ }^{21}$ sion parameter, the more clustered the group is. The ${ }^{22}$ offspring distribution (5) yields the following: 23

${ }_{26}^{24} \quad G_{Z_{1}}(x)=\left(\frac{s}{s+(1-x) c \sqrt{\lambda}}\right)^{s}$.

27

${ }_{28}$ When $|x|<1+s / c \sqrt{\lambda}$; otherwise, the value is zero.

29 To show the effect of the vector-bias in the extinc${ }^{30}$ tion of malaria, we calculated the extinction probabil${ }^{31}$ ity using the varying bias parameter $\lambda$ and dispersion ${ }^{32}$ parameter $s$. In many cases, it is difficult to find the ${ }^{33}$ solution of (4) analytically. Hence, we used a numeri${ }^{34} \mathrm{cal}$ method known as cobwebbing for finding the fixed ${ }^{35}$ point of $G_{Z_{1}}$ in $(7)$. We performed iterations using the ${ }^{36}$ initial assumption $x_{1}=0.1$, until the difference of the ${ }^{37}$ prior and posterior term, $\left|x_{\text {prior }}-x_{\text {posterior }}\right|<10^{-10}$ ${ }^{38}$ was reached. Because negative binomial distributions ${ }^{39}$ with $s=1000$ and $s \rightarrow \infty$ are indistinguishable in practice [43], it is sufficient to simulate the model us- ${ }^{18}$ ing $s \in(0.001,1000)$.

Results

In this section, we investigated the impact of vector- ${ }^{-23}$ bias on malaria invasion and extinction under a low- 24 transmission setting. The parameter values adopted in ${ }_{25}$ the simulation are listed in Table 2 .

The probability of mosquito infection from infectious 28 human

We showed the effect of vector-bias on the mosquito ${ }^{30}$ infections. If we let $X$ be an event in which a human ${ }^{31}$ is infectious and is chosen by a mosquito, then the $\mathrm{e}^{32}$ probability that a human is infectious when chosen by ${ }^{33}$ a mosquito, $\mathbb{P}(X)$ was given by $\frac{\lambda \mathcal{I}_{h}}{1+(\lambda-1) \mathcal{I}_{h}}$ from the ${ }^{34}$ underlying model (1), where $\mathcal{I}_{h}=\frac{I_{h}}{N}$. In this way, if 35 we set a fixed condition $\mathcal{I}_{h}=k$ and use the probability ${ }^{36}$ density of bias parameter $\lambda$, i.e., Equation (2), we can ${ }^{37}$ obtain the probability that a human is infectious when ${ }^{38}$ chosen by a mosquito under a fixed infectious condition ${ }^{39}$ 
1 Table 2 Parameter descriptions and values.

\begin{tabular}{llll}
\hline 2 & Description, Notation (Unit) & Value & Source \\
\hline 3 & Human birth rate and death rate, & $3.90 \times 10^{-5}$ & Estimated \\
4 & & \\
\hline
\end{tabular}

5 Mosquito birth rate and death 0.10 [27] rate, $\mu_{v}\left(\right.$ day $\left.^{-1}\right)$

Biting rate of a mosquito, $b \quad 0.35 \quad$ [27]

$7\left(\right.$ day $\left.^{-1}\right)$

8 Per capita recovery rate, $\gamma \quad 3.50 \times 10^{-3} \quad$ [27]

$9\left(\right.$ day $\left.^{-1}\right)$

10 Per capita rate of loss of immu- $2.74 \times 10^{-3} \quad$ [27]

11 nity, $\xi\left(\right.$ day $\left.^{-1}\right)$

12 Transmission probability of infec- 0.024

tion from an infectious mosquito

to a non-malaria infected human

14 when a contact between the two

15 occurs, $p_{h}(1)$

16 Transmission probability of infec- 0.24

[27]

17 tion from an infectious human to

a susceptible mosquito when a

18 contact between the two occurs,

$19 p_{v}(1)$

20

21

${ }_{22} \mathcal{I}_{h}=k$ as

23

23

24

$\mathbb{P}\left(X \mid \mathcal{I}_{h}=x\right)=\int_{0}^{\infty} \mathbb{P}\left(X \mid \lambda=\varphi, \mathcal{I}_{h}=x\right) f_{\lambda}(\varphi) \mathrm{d} \varphi$

25

26 Figure 3 shows the probability of the rate of infec${ }^{27}$ tious humans $\mathcal{I}_{h}$. The solid line indicates the probabil${ }^{28}$ ity $\mathbb{P}\left(X \mid \mathcal{I}_{h}=x\right)$ without considering the vector-bias, ${ }^{29}$ and the dashed line shows the probability when con${ }^{30}$ sidering the vector-bias for a fixed $x$. In the figure,

${ }^{31}$ the probability that a human is infectious when cho${ }^{32}$ sen by a mosquito is always higher when the vector${ }^{33}$ bias is considered than when it is not. For example, ${ }^{34}$ a mosquito might bite an infectious human once after ${ }^{35}$ two attempts, despite infectious humans in the commu${ }^{36}$ nity being approximately $25 \%$. Thus, mosquitoes, par${ }^{37}$ ticularly susceptible mosquitoes, have a greater chance ${ }^{38}$ of being infected with malaria owing to a vector-bias ${ }^{39}$ in the invading situation.
Figure 3 The probability that the human is infectious when chosen by a mosquito under the fixed condition $\mathcal{I}_{h}=x$ when not considering (solid line) and considering (dashed line) a vector-bias effect.

These results indicate that the vector-bias first in- 6 creases the probability of a mosquito being infected ${ }^{7}$ from the first infectious human infection from the first ${ }^{8}$ infectious human during an invasion, leading to a rapid $^{9}$ increase in infectious mosquitos, which finally results ${ }^{10}$ in an easy invasion of human populations by infectious ${ }^{11}$ mosquitoes, as compared to an invasion in the absence ${ }^{12}$ of such bias. By contrast, after an invasion of malaria, ${ }^{13}$ the probability of an infectious mosquito biting into ${ }^{14}$ a susceptible human decreases as the fraction of infec- ${ }^{15}$ tious humans $x$ increases. Thus, we may say that, in $\mathrm{a}^{16}$ highly endemic area, we might under-estimate the risk ${ }^{17}$ of infection unless the vector-bias is considered. This ${ }^{18}$ supports previous the results in [27], which show that ${ }^{19}$ the effect lessens the endemicity in a high transmission ${ }^{20}$ area and increases it in a low transmission area. $\quad 21$

Invasion of malaria

We simulated an individual-based CTMC model to24 show the effect of vector-bias in the early invasive 25 phase after a first outbreak. All simulations are re 26 peated $10^{4}$ times under the same qualifications. We as 27 sume that the populations of humans and mosquitoes 28 are same, at 1000, i.e., $N=M=10^{3}$. The results were 29 compared with the simulations wherein the vector-bias 30 was not considered. In addition, we compared the two ${ }^{31}$ invasion scenarios:

(Scenario A) invasion from a mosquito, i.e., 33 $\left(I_{h}(0), I_{v}(0)\right)=(0,1) ;$ and $\quad 34$

(Scenario B) invasion from a human, i.e., 35

$$
\left(I_{h}(0), I_{v}(0)\right)=(1,0) . \quad 36
$$

In addition, $R_{h}(0)=0$ was assumed. $\quad 37$

Table 3 shows the probability that an infection will ${ }^{38}$ occur, and the probability of a secondary infection of ${ }^{39}$ 
Table 3 Probabilities of the secondary infection and time to the secondary infection against two initial invasion scenarios.

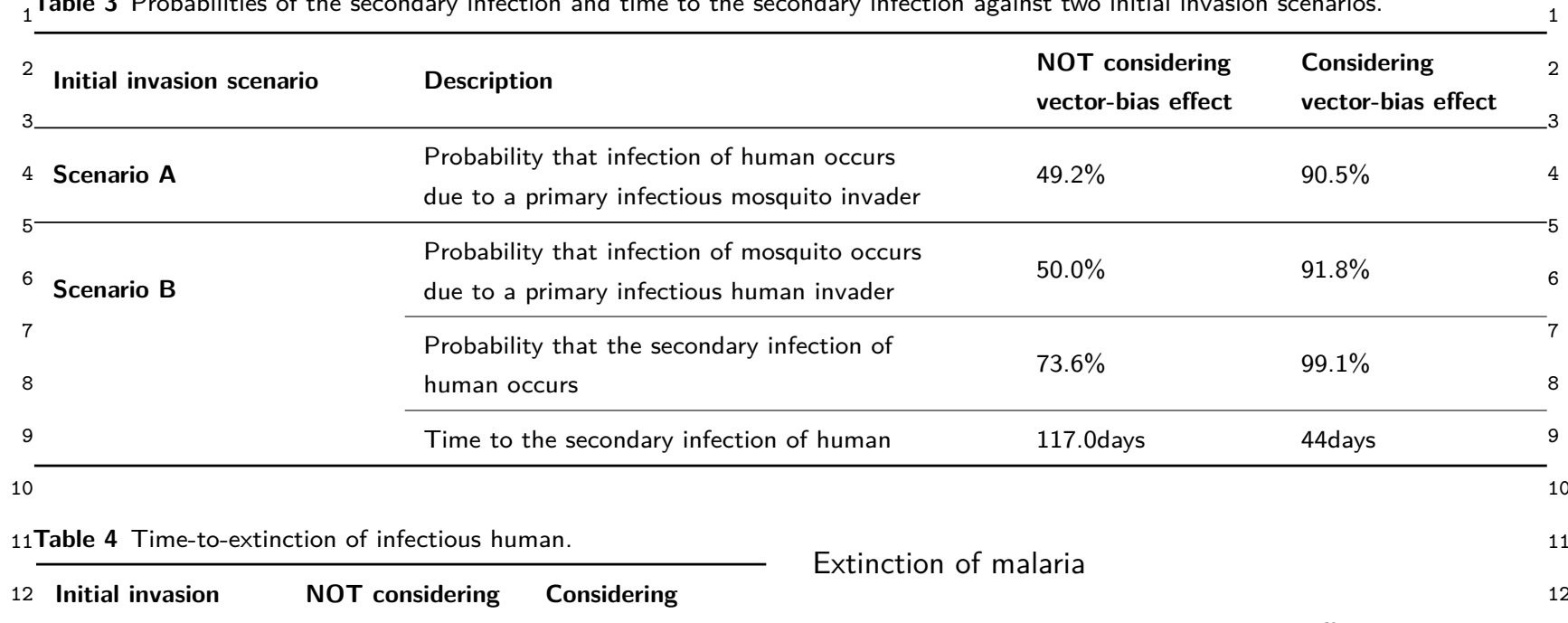

Figure 5 and Figure 6 show the offspring distribu- ${ }_{13}$ tion of secondary infection and corresponding extinc- ${ }_{14}$ tion probabilities under various dispersion scenarios $_{15}$ respectively. The left panel of Figure 6 indicates the ${ }_{16}$ extinction probabilities against the dispersion parame- ${ }_{-17}$ ${ }_{18}$ mosquitos and humans, as two invasion scenarios [46] ${ }_{19}$ and the time taken for the secondary infection of a hu${ }_{20}$ man to occur, under the human invasion scenario. In ${ }_{21}$ both cases, the probability of infection is doubled when ${ }_{22}$ considering the vector-bias. When an infection occurs, ${ }_{23}$ the probability that malaria will persist increases due ${ }_{24}$ to the vector-bias. This is because the number of in${ }_{25}$ fected people increases after the first invader arrives, ${ }_{26}$ whether a mosquito or human. When there is an inva${ }_{27}$ sion by one person, vector-bias has a three-fold impact ${ }_{28}$ on the results. Therefore, it is important to apply treat${ }_{29}$ ment prior to the invasion because the probability of 3opersistence increases rapidly, once the initial invasion ${ }_{31}$ occurs.

32 Figure 4 shows the endemic state distribution when ${ }^{33}$ eliminating the states equal to zero and the invasion ${ }^{34}$ probabilities, defined as the probability of an endemic ${ }^{35}$ by a primary invader, under both scenarios. The en${ }^{36}$ demic prevalence of humans and mosquitos is high for ${ }^{37}$ both, when considering vector-bias. In addition, the ${ }^{38}$ probability of an endemic is twice as high as when not ${ }^{39}$ considering vector-bias under both scenarios. ter. The extinction probability decreases as the disper-$^{-18}$ sion parameter increases, because the heterogeneity in- ${ }_{19}$ creases the uncertainty of infection and consequently $y_{20}$ increases the extinction probability. In addition, for ${ }_{21}$ a fixed dispersion parameter $s$, the extinction proba- 22 bility decreases when considering the vector-bias $\mathrm{as}_{23}$ based on the expression for $R_{0}$, we know that an in- ${ }_{24}$ creasing $\lambda$ increases $R_{0}$. Therefore, an increase in $\lambda$ will $_{25}$ also reduce the disease extinction probability. Thus, $i_{26}$ can be seen that the vector-bias reduces the chance of $f_{27}$ malaria extinction in some endemic or invasive commu- $_{28}$ nities. Although vector-bias lessens the endemic level ${ }_{29}$ in a high transmission area [27], it provides a positive ${ }_{30}$ potential to maintain the malaria endemic. 31

The right panel of Figure 6 shows that the vector ${ }^{32}$ bias has no effect on reducing the malaria extinction ${ }^{33}$ probability in a highly heterogeneous environment. $\mathrm{Al}^{-34}$ though the bias towards infectious humans is 20 -times $^{35}$ higher, the extinction probability is only reduced to ${ }^{36}$ $0.23 \%$ (0.9993 to 0.9970$)$. However, as heterogeneity ${ }^{37}$ decreases, the decreasing rate of extinction probabil 38 ity owing to vector-bias increases. It can be seen that ${ }^{39}$ 
Figure 4 Multivariate distribution of endemic states (states at 10 years) when removing states hitting zero in Scenario A (left) and

Scenario B (right). The invasion probability is abbreviated as IP. Northern and southern curves of each panels are histograms of endemic states against infectious humans and infectious mosquitoes, respectively.

4

Figure 5 Offspring distribution (6) of secondary infections through negative binomial counting model

Figure 6 Extinction probability against dispersion parameter $s$ (left) and bias parameter $\lambda$ (right).

${ }^{10}$ the extinction probability decreases rapidly as the bias ${ }^{11}$ parameter increases but remains above a certain level.

${ }^{12}$ In terms of the adaptive host manipulation hypothesis

${ }^{13}$ in biology, these results suggest two possibilities: first,

${ }^{14}$ an extreme-biased behaviour of mosquitos can occur

${ }^{15}$ in areas having a high heterogeneity to increase the vi-

${ }^{16}$ ability of a malaria parasite, and second, a low level of

${ }^{17}$ biased behaviour is sufficient to increase the viability

${ }^{18}$ of the parasite in areas with a low heterogeneity.

19 Table 4 shows the time-to-extinction through the

${ }^{20}$ individual-based CTMC model. The time is short

${ }^{21}$ when considering a vector-bias, which means that if

${ }^{22}$ it does not occur during an early phase, and does not

${ }^{23}$ become extinct, it becomes an invasion with a high

${ }^{24}$ probability. This is because the vector-bias accelerates

${ }^{25}$ the initial invasion speed owing to an increase in $R_{0}$,

${ }^{26}$ which makes the extinction more difficult, by rapidly

${ }^{27}$ making the endemicity exceed the extinction threshold

${ }^{28}$ and increasing the extinction probability.

29

\section{${ }^{30}$ Conclusion}

${ }^{31}$ The biased behaviour of mosquitos toward infectious

${ }^{32}$ humans accelerates an invasion of malaria into humans

${ }^{33}$ to endemic levels within a short time span, and during

${ }^{34}$ an endemic state, it makes the eradication of malaria

${ }^{35}$ difficult by lowering the extinction probability. There-

${ }^{36}$ fore, in areas without malaria, it is most important not

${ }^{37}$ to let the first patient enter a community, without a

${ }^{38}$ thorough examination, and even if an outbreak starts

${ }^{39}$ from the first patient, it is strongly necessary to stop the malaria endemic by introducing control measures ${ }^{10}$ in the earliest possible time. The results of this study ${ }^{11}$ also indicate that an extremely biased behaviour may ${ }^{12}$ occur in a highly heterogeneous environment, whereas ${ }^{13}$ a biased behaviour may not be necessary for the sur-14 vival of parasites in a low heterogeneous environment ${ }^{15}$ from an evolution perspective. In the future, malaria ${ }^{16}$ will evolve into more diverse areas owing to the rapid ${ }^{17}$ climate change. Therefore, studies on observing the be-18 haviour of mosquitoes and identifying the causes of ${ }^{19}$ such behavioural changes are needed for the predic- ${ }^{20}$ tion and control of malaria. In addition, studies on ${ }^{21}$ malaria transmission dynamics by considering the ma-22 nipulated behaviours and evolution of parasites should ${ }^{23}$ be actively carried out for the eradication of malaria. ${ }^{24}$

Ethics approval and consent to participate

Not applicable.

Consent for publication

Not applicable.

Availability of data and materials

All data generated or analysed during this study are included in published 32 articles [12] and [27].

Competing interests

The authors declare that they have no competing interests.

This work was supported by the National Research Foundation of Korea 37 (NRF) Grant funded by the Korean Government (MSIP)

NRF-2017R1A5A1015722 to II Hyo Jung; NRF-2017R1E1A1A03069992 to 38 Byul Nim Kim; NRF-2019R1A2C2007249 to II Hyo Jung. 
${ }^{1}$ Author's contributions

$2 \mathrm{YK}$ and $\mathrm{IHJ}$ conceptualized the roles of the group. MAM and IHJ designed

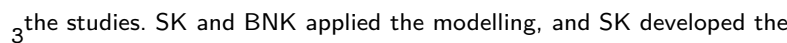
numerical simulation. SK, MAM, and YK verified the results. All the

members discussed the results and wrote the manuscript.

5

Acknowledgements

${ }^{6}$ Not applicable.

${ }^{7}$ Author details

$8^{1}$ Department of Mathematics, Pusan National University, 2,

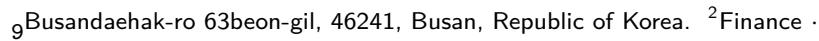
Fishery - Manufacture Industrial Mathematics Center on Big Data, Pusan

${ }^{10}$ National University, 2, Busandaehak-ro 63beon-gil, 46241, Busan, Republic 11 of Korea. ${ }^{3}$ Department of Mathematics, Kyungpook National University, $12{ }^{80}$, Daehak-ro, 41566, Daegu, Republic of Korea. ${ }^{4}$ Institute for

Mathematical Convergence, Kyungpook National University, 80, Daehak-ro, ${ }^{13} 41566$, Daegu, Republic of Korea. ${ }^{5}$ Department of Mathematics and 14Physics, North South University, Bashundhara, 1229, Dhaka, Bangladesh.

\section{${ }^{15}$ References}

16 1. Holmes JC, Bethel WM, Canning EU, Wright CA. Behavioural aspects of parasite transmission. Zoological Journal of the Linnean Society. 1972;51:123-149.

18 2. Thomas F, Adamo S, Moore J. Parasitic manipulation: where are we

19 and where should we go? Behavioural processes. 2005;68(3):185-199.

3. Braks MAH, Anderson RA, Knols BGJ. Infochemicals in mosquito host selection: Human skin microflora and Plasmodium parasites; 1999.

21 4. Zwiebel LJ, Takken W. Olfactory regulation of mosquito-host

22 interactions. Insect Biochemistry and Molecular Biology. 2004;34(7):645-652.

5. Clements AN. The biology of mosquitoes. Vol. 2. Sensory reception 24 and behaviour; 1999

256 . Nacher M. Charming the mosquito: do malaria symptoms increase the

26 attractiveness of the host for the vector? Medical Hypotheses. 2005;64(4):788-791.

27 7. Willem T, Bart GJK. Olfaction in vector-host interactions: Ecology

28 and control of vector-borne diseases. pp 351. vol. 2. P.O. Box 220,

6700 AE Wageningen, The Netherlands: Wageningen Academic Publishers; 2010.

30 8. Day JF, Ebert KM, Edman JD. Feeding patterns of mosquitoes

31 simultaneously exposed to malarious and healthy mice, including a method for separating blood meals from conspecific hosts. Journal of Medical Entomology. 1983;20:120-127.

33 9. Day JF, Edman JD. Malaria renders mice susceptible to mosquito

34 feeding when gametocytes are most infective. Journal of Parasitology. 1983;69:163-170

10. Mahon R, Gibbs A. Arbovirus-infected hens attract more mosquitoes

36 JD Mackenzie, ed Viral disease in Southeast Asia and the western

37 Pacific Academic Press, Sydney. 1982;p. 502-504

11. Turell MJ, Bailey CL, Rossi CA. Increased mosquito feeding on Rift Valley fever virus-infected lambs. The American Journal of Tropical

39 Medicine and Hygiene. 1985;33:1232-1238.
12. Lacroix R, Mukabana WR, Gouagna LC, Koella JC. Malaria Infection ${ }^{1}$ Increases Attractiveness of Humans to Mosquitoes. PLoS Biol. 2 2005;3(9):e298.

13. Chamchod F, Britton NF. Analysis of a vector-bias model on malaria transmission. Bulletin of mathematical biology. 2011;73(3):639-657. 4

14. Koella JC, Packer MJ. Malaria parasites enhance blood-feeding of 5 their naturally infected vector Anopheles punctulatus. Parasitology. 6 1996;113(02):105-109

15. Scott TW, Takken W. Feeding strategies of anthropophilic mosquitoes ${ }^{7}$ result in increased risk of pathogen transmission. Trends in 8 Parasitology. 2012;28(3):114-121.

16. Cornet S, Nicot A, Rivero A, Gandon S. Malaria infection increases bird attractiveness to uninfected mosquitoes. Ecology letters. 2013;16(3):323-329

17. De Moraes CM, Stanczyk NM, Betz HS, Pulido H, Sim DG, Read AF, 12 et al. Malaria-induced changes in host odors enhance mosquito attraction. Proceedings of the National Academy of Sciences. 2014;111(30):11079-11084.

18. Busula AO, Bousema T, Mweresa CK, Masiga D, Logan JG, Sauerwein ${ }_{15}$ RW, et al. Gametocytemia and attractiveness of Plasmodium falciparum-infected Kenyan children to Anopheles gambiae mosquitoes. ${ }^{16}$ The Journal of Infectious Diseases. 2017;216(3):291-295. 17

19. Busula AO, Verhulst NO, Bousema T, Takken W, de Boer JG. 18 Mechanisms of Plasmodium-enhanced attraction of mosquito vectors. Trends in parasitology. 2017;33(12):961-973.

20. Sherrard-Smith E, Skarp JE, Beale AD, Fornadel C, Norris LC, Moore 20 $\mathrm{SJ}$, et al. Mosquito feeding behavior and how it influences residual 21 malaria transmission across Africa. Proceedings of the National Academy of Sciences. 2019;116(30):15086-15095

21. Suh E, Grossman MK, Waite JL, Dennington NL, Sherrard-Smith E, 23 Churcher TS, et al. The influence of feeding behaviour and temperature on the capacity of mosquitoes to transmit malaria. Nature Ecology \& Evolution. 2020;p. 1-12.

22. Kingsolver JG. Mosquito host choice and the epidemiology of malaria. 26 The American Naturalist. 1987;130:811-827.

23. Agusto FB, Tchuenche JM. Control strategies for the spread of malaria in humans with variable attractiveness. Mathematical 28 Population Studies. 2013;20(2):82-100.

24. Buonomo B, Vargas-De-León C. Stability and bifurcation analysis of a 30 vector-bias model of malaria transmission. Mathematical Biosciences. 2013;242(1):59-67.

25. Cator LJ, Lynch PA, Read AF, Thomas MB. Do malaria parasites 32 manipulate mosquitoes? Trends in parasitology. 2012;28(11):466-470. 33

26. Cator LJ, Lynch PA, Thomas MB, Read AF. Alterations in mosquito behaviour by malaria parasites: Potential impact on force of infection. ${ }^{34}$ Malaria Journal. 2014;

27. Kim S, Masud MA, Cho G, Jung IH. Analysis of a vector-bias effect in ${ }_{36}$ the spread of malaria between two different incidence areas. Journal of Theoretical Biology. 2017;

28. Wang $\mathrm{X}$, Zhao $\mathrm{XQ}$. A periodic vector-bias malaria model with 38 incubation period. SIAM Journal on Applied Mathematics.

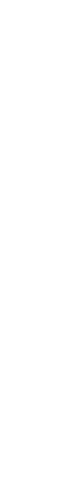

10

1

3

5

7

8

9

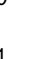

2



probability and endemic prevalence for Markovian SIS dynamics on

229. Li J, Teng Z, Zhang L. Stability and bifurcation in a vector-bias model networks. PloS one. 2013;8(7):e69028. 2

3 of malaria transmission with delay. Mathematics and Computers in Simulation. 2018;152:15-34.

40. Pan F, Cui X, Xue D, Lu Z. Stability analysis of a fractional-order

5 vector-bias model on malaria transmission. In: 2019 Chinese Control

And Decision Conference (CCDC). IEEE; 2019. p. 6363-6367.

31. Wang J, Chen Y. Threshold dynamics of a vector-borne disease model 7 with spatial structure and vector-bias. Applied Mathematics Letters. 8 2020;100:106052.

9 32. Mojeeb AL, Li J. Analysis of a vector-bias malaria transmission model with application to Mexico, Sudan and Democratic Republic of the

10 Congo. Journal of Theoretical Biology. 2019;464:72-84.

1133. Jacquez JA, O'Neill P. Reproduction numbers and thresholds in

12 stochastic epidemic models I. Homogeneous populations. Mathematical Biosciences. 1991;107(2):161-186.

${ }^{13} 34$. Britton T. Stochastic epidemic models: a survey. Mathematical

14 biosciences. 2010;225(1):24-35.

15 35. Gillespie DT. Exact stochastic simulation of coupled chemical reactions. The Journal of Physical Chemistry. 1977;81(25):2340-2361.

${ }^{16}$ 36. Gillespie DT. Stochastic simulation of chemical kinetics. Annu Rev

17 Phys Chem. 2007;58:35-55.

18 37. Higham DJ. Modeling and simulating chemical reactions. SIAM Review. 2008;.

19 38. Diekmann O, Heesterbeek JAP, Metz JAJ. On the definition and the

20 computation of the basic reproduction ratio $\mathrm{R} 0$ in models for infectious

22 39. Raphaka K, Sánchez-Molano E, Tsairidou S, Anacleto O, Glass EJ,

23 Woolliams JA, et al. Impact of genetic selection for increased cattle

24 resistance to bovine tuberculosis on disease transmission dynamics. Frontiers in veterinary science. 2018;5.

${ }^{25}$ 40. Pemberton-Ross P, Chitnis N, Pothin E, Smith TA. A stochastic

26 model for the probability of malaria extinction by mass drug

27 administration. Malaria journal. 2017;16(1):376

41. Haccou $P$, Haccou $P$, Jagers $P$, Vatutin VA, Vatutin VA. Branching 28 processes: variation, growth, and extinction of populations. 5.

29 Cambridge university press; 2005.

30 42. Lloyd-Smith JO, Schreiber SJ, Kopp PE, Getz WM. Superspreading and the effect of individual variation on disease emergence. Nature. 312005

3243. Lloyd-Smith JO. Maximum likelihood estimation of the negative

33 binomial dispersion parameter for highly overdispersed data, with applications to infectious diseases. PloS one. 2007;2(2):e180.

${ }^{34} 44$. Hogg RV, McKean J, Craig AT. Introduction to Mathematical

35 Statistics. 7th ed. Pearson; 2012.

36 45. Meehan MT, Cope RC, McBryde ES. On the probability of strain invasion in endemic settings: Accounting for individual heterogeneity 37 and control in multi-strain dynamics. Journal of theoretical biology. $38 \quad 2020 ; 487: 110109$ 


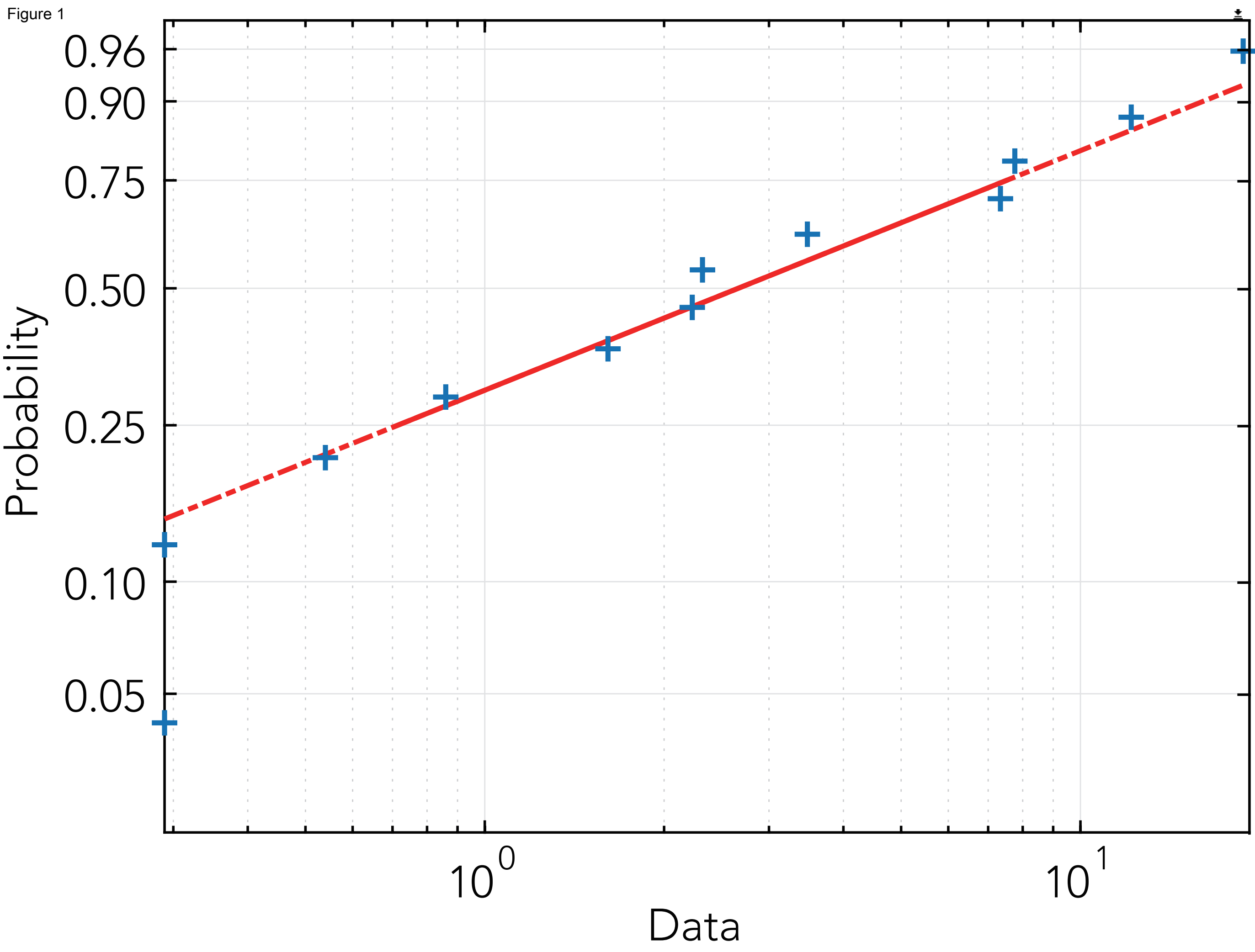




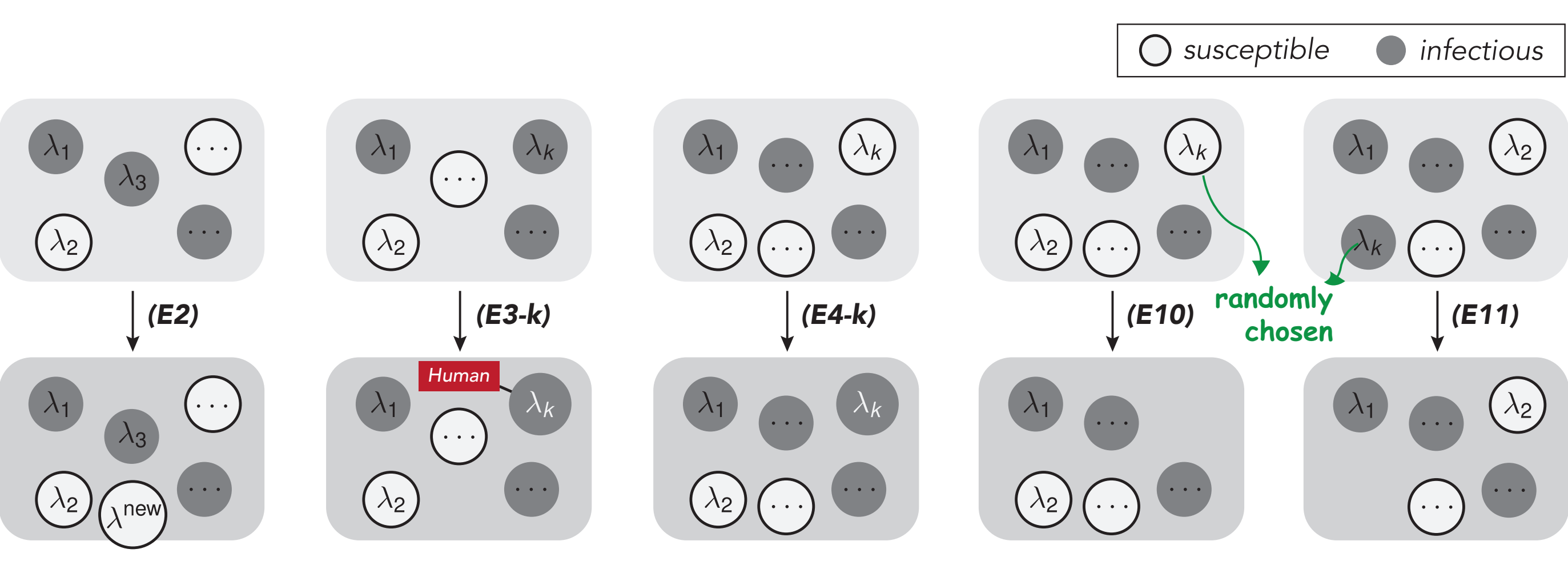




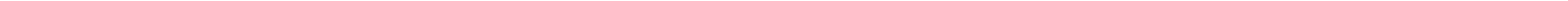




\section{Scenario A}

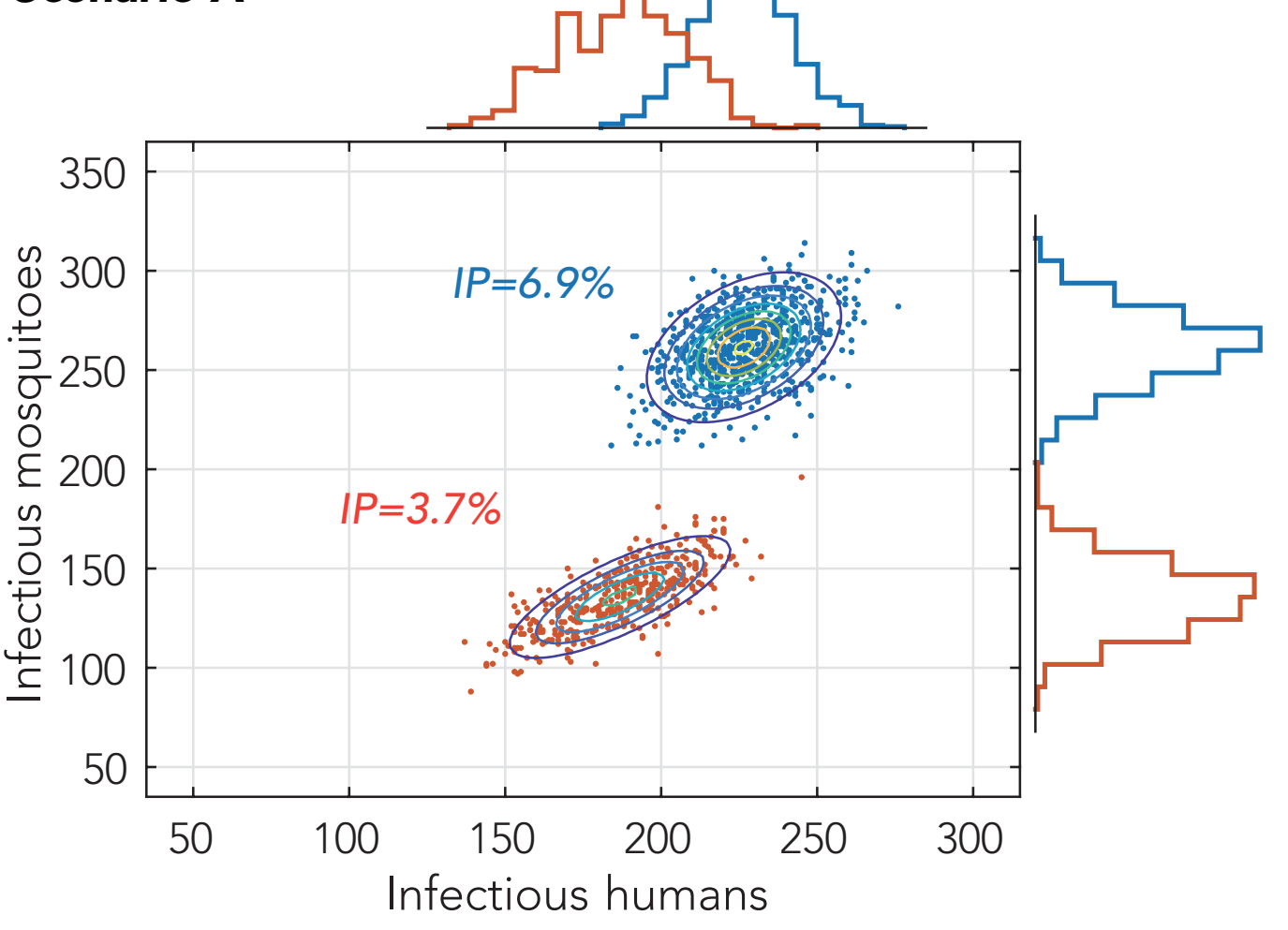

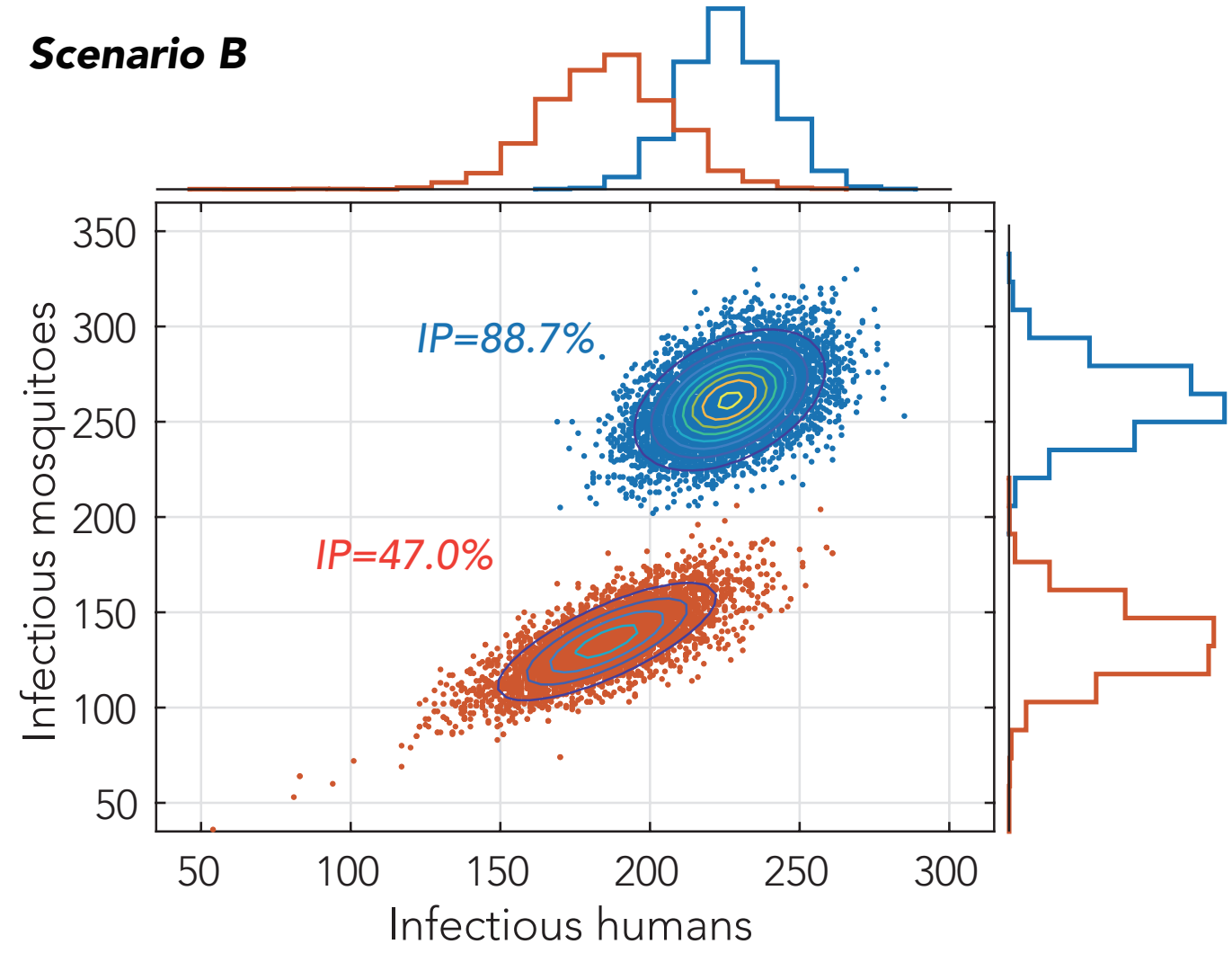

- considering vector-bias effect

- not considering vector-bias effect

D contours of multivariate Gaussian distribution fit 

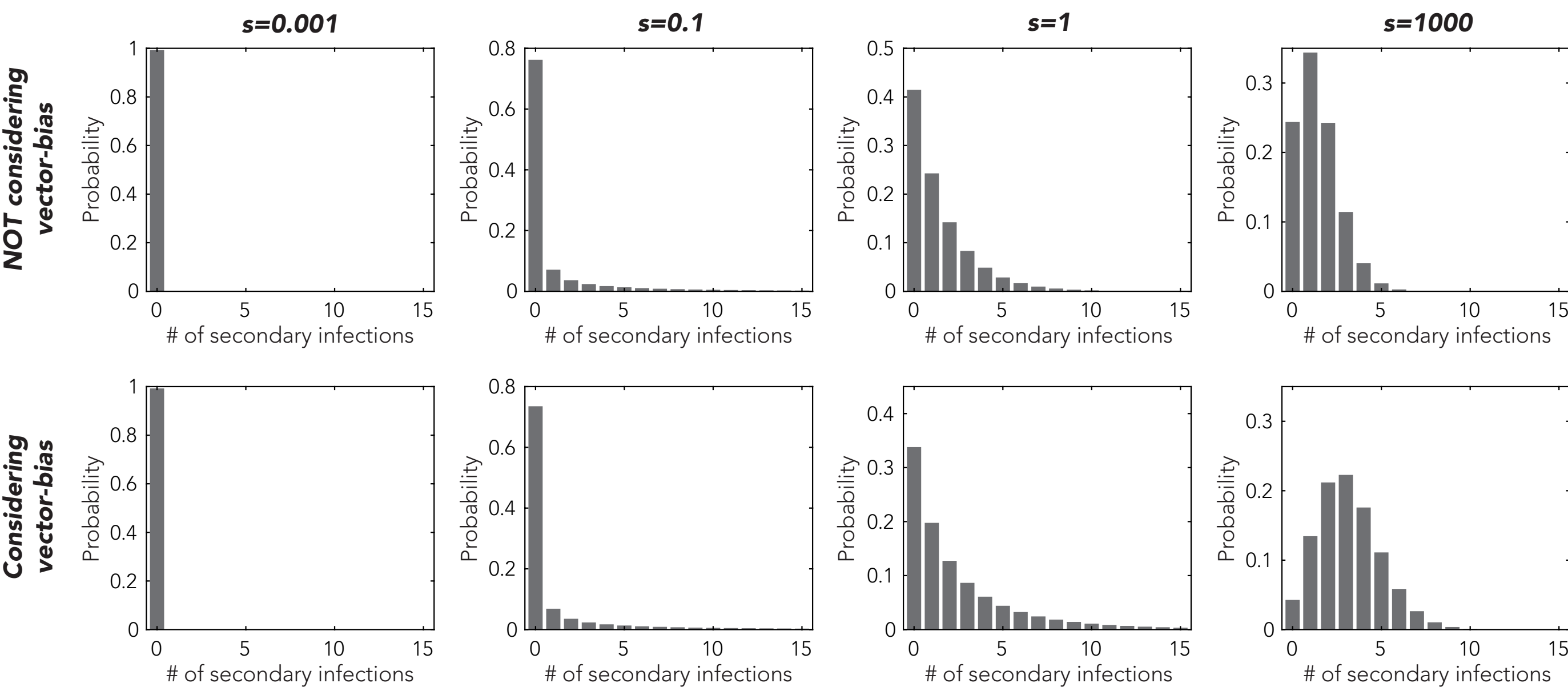

\section{Heterogeneity}

Homogeneous (Time stationary) 

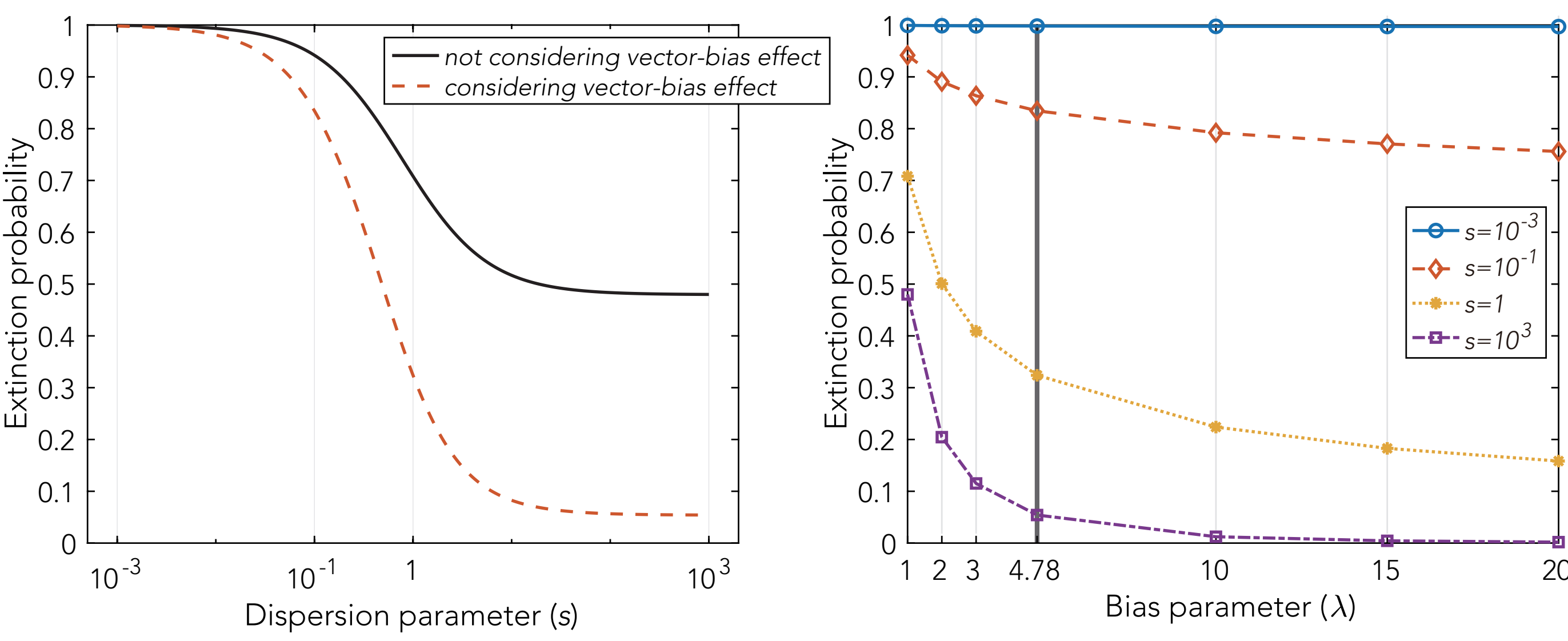
Figures

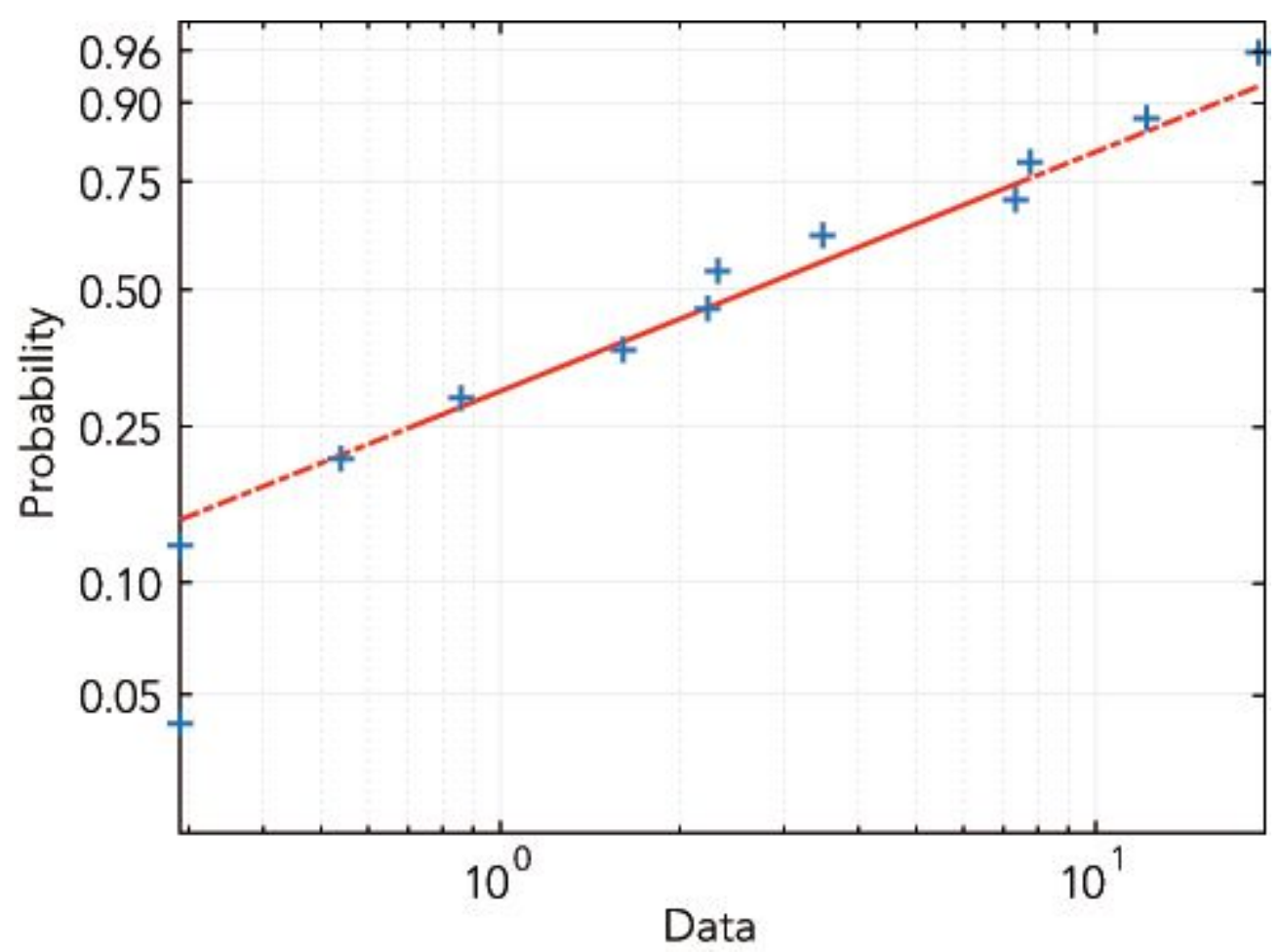

Figure 1

Weibull probability plot of bias parameter: the probability density function of Weibull distribution is given by (2) with $\alpha=4: 34$ and $\beta=0: 83$.

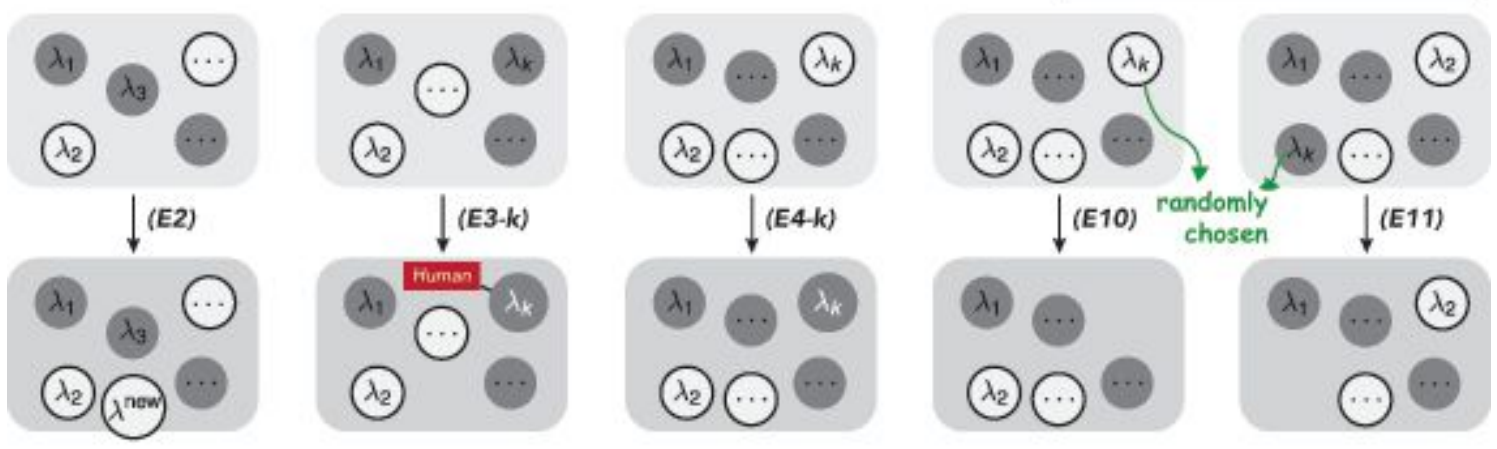

Figure 2

Description of the individual-based CTMC model and its simulation algorithm. 


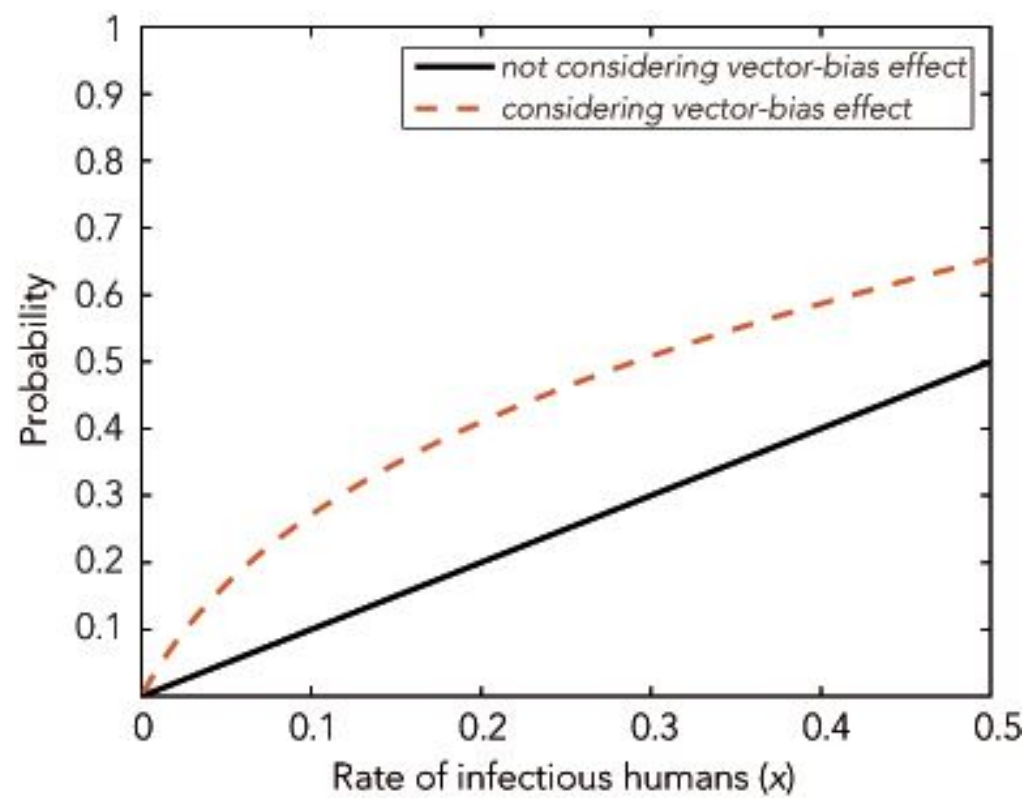

Figure 3

The probability that the human is infectious when chosen by a mosquito under the fixed condition $\mathrm{lh}=\mathrm{x}$ when not considering (solid line) and considering (dashed line) a vector-bias effect.
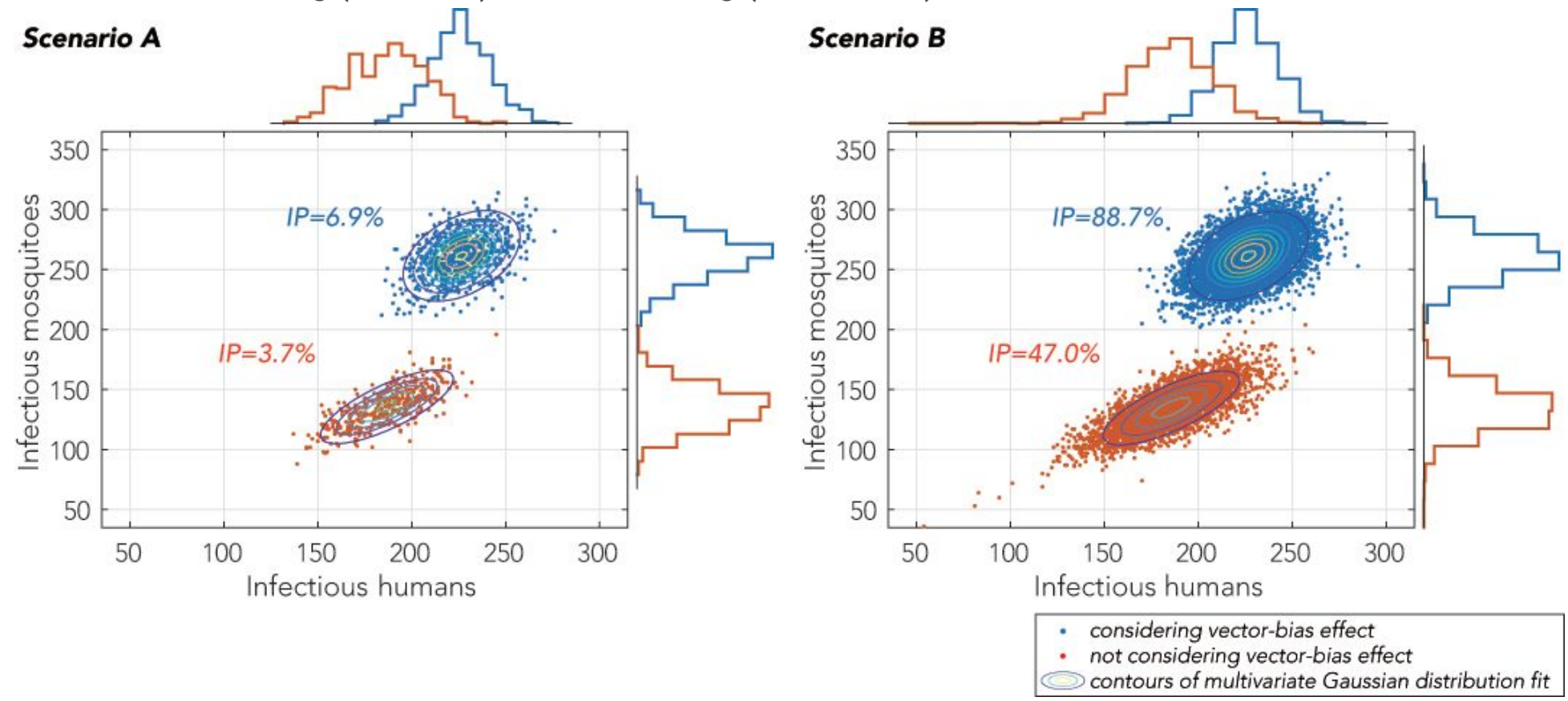

Figure 4

Multivariate distribution of endemic states (states at 10 years) when removing states hitting zero in Scenario A (left) and Scenario B (right). The invasion probability is abbreviated as IP. Northern and southern curves of each panels are histograms of endemic states against infectious humans and infectious mosquitoes, respectively. 

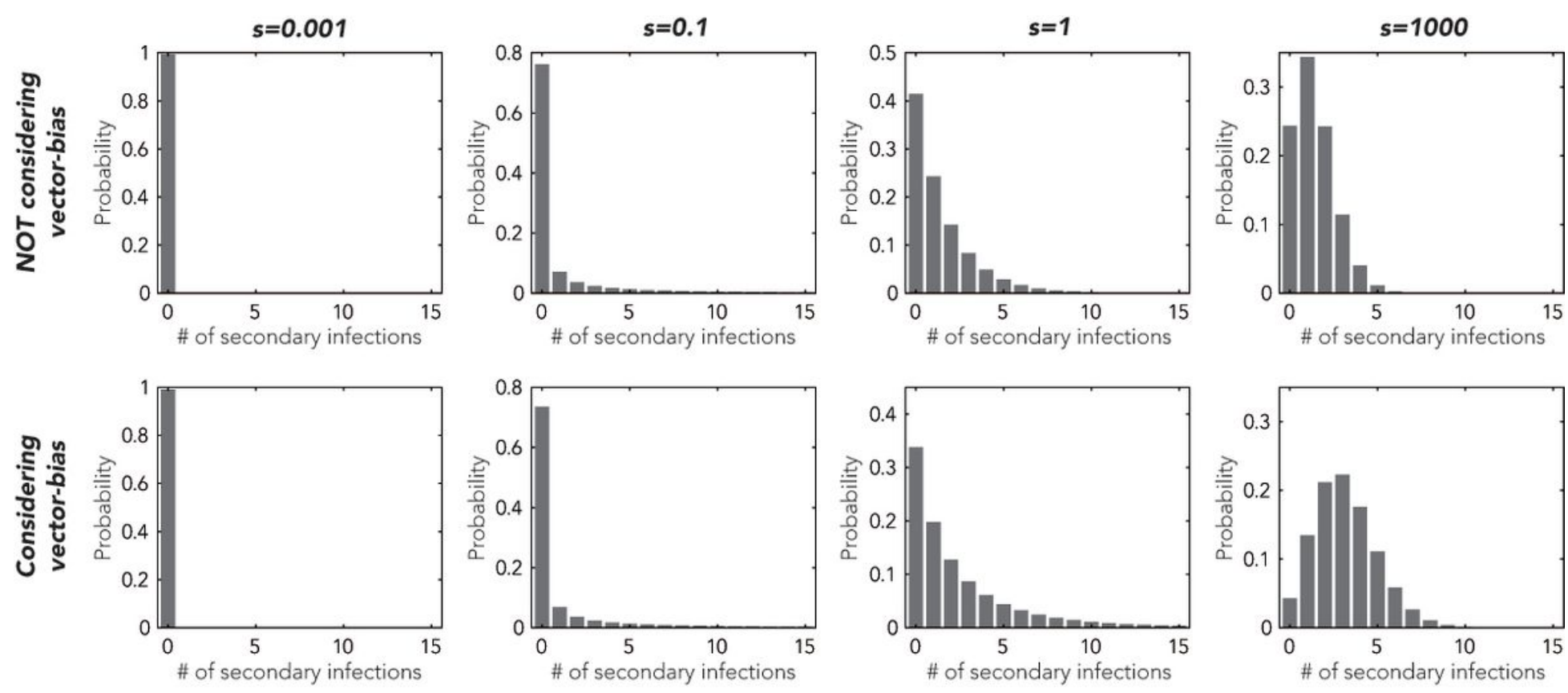

Heterogeneity

Homogeneous (Time stationary)

\section{Figure 5}

Offspring distribution (6) of secondary infections through negative binomial counting model
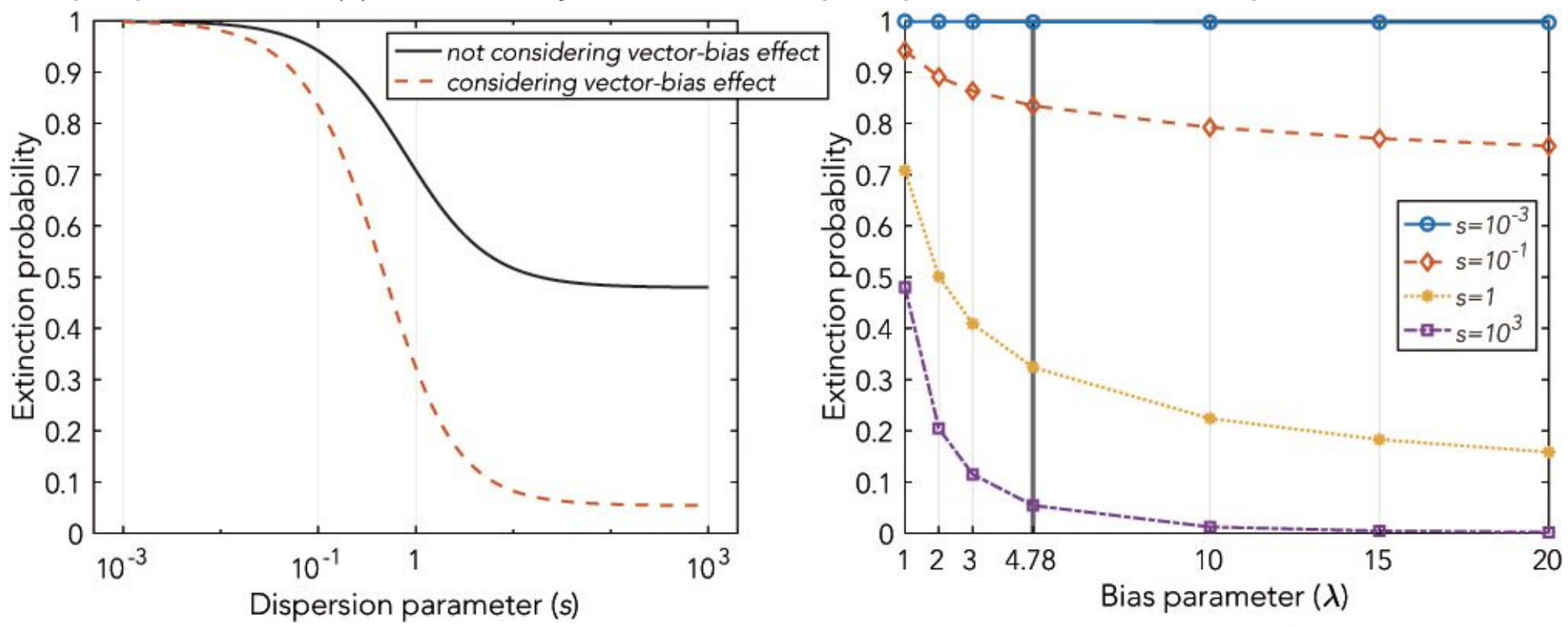

\section{Figure 6}

Extinction probability against dispersion parameter $s$ (left) and bias parameter $\lambda$ (right). 